\title{
Pancreatic hydatid cyst mimicking a choledochal cyst
}

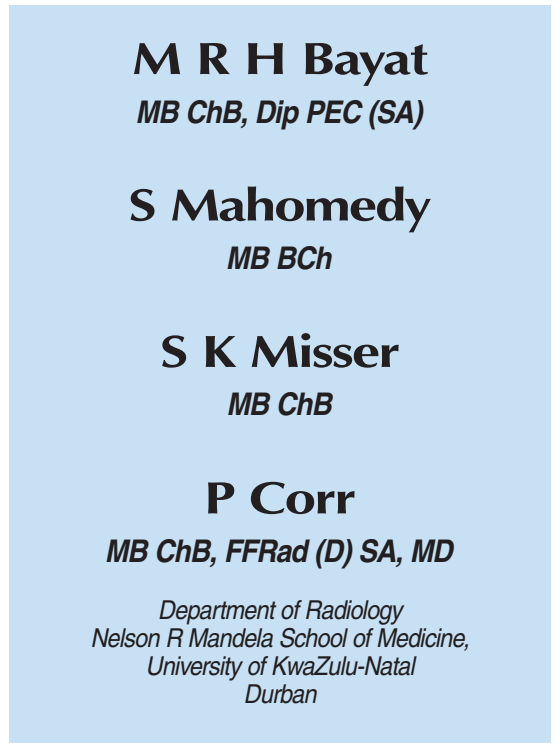

\section{Abstract}

Hydatid disease is endemic to South Africa. Radiological findings may help establish the diagnosis and aid preoperative preparation. Unusual locations and atypical imaging appearances may complicate the differential diagnosis. A case of isolated hydatid disease mimicking choledochal cyst is presented. Primary hydatid disease of the pancreas in the paediatric population is rare and this is only the sixth case reported in the English literature. This article highlights the importance of always considering hydatid disease in our patient population whenever a cystic mass of the pancreas is identified.

\section{Introduction}

Hydatid disease is a parasitic infection caused by the larval stage of the tapeworm Echinococcus. Human infection is endemic in sheep-raising areas of the world, including South Africa. Pancreatic involvement in adults occurs with a reported prevalence of $0.25 \% .{ }^{1}$ Primary pancreatic hydatid disease in childhood with no other associated visceral involvement is extremely rare, and only 5 cases have been reported to date. ${ }^{2-6}$ Nonetheless, hydatid disease should always be included in the differential diagnosis when a cystic lesion is identified in a patient from an endemic area.

\section{Case report}

An 11-year-old girl was admitted with symptoms of epigastric pain and vomiting. Examination revealed a palpable epigastric mass and jaundice. The latter was confirmed biochemically. The serum amylase was normal. Abdominal ultrasound (Fig. 1a and b) and computed tomography (CT) scan (Fig. 2a and b) of the abdomen were obtained.

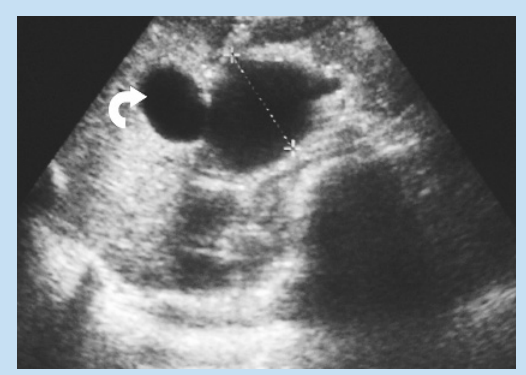

Fig.1a. Coronal ultrasound through the liver and common bile duct. The dilated bile duct (curved arrow) appears to communicate with a large anechoic cystic collection in the pancreatic head.

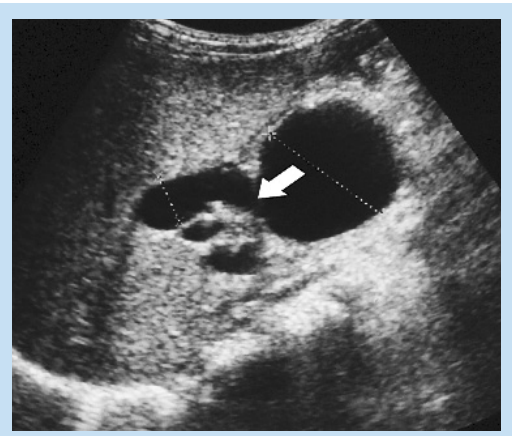

Fig.1b. Coronal ultrasound through the liver and common bile duct. The wall separating the two structures (arrow) is now visualised but is thin and almost imperceptible leading to diagnostic confusion.

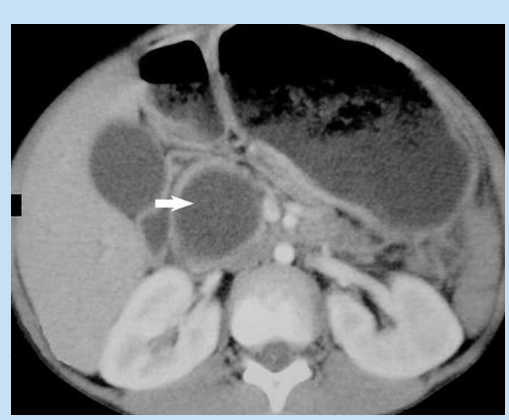

Fig. 2a. Axial enhanced CT demonstrates a 4 $\times 2 \mathrm{~cm}$ type 1 hyatid cyst in the pancreas (arrow). The dilated debris-filled stomach and compressed second part of the duodenum are also seen.

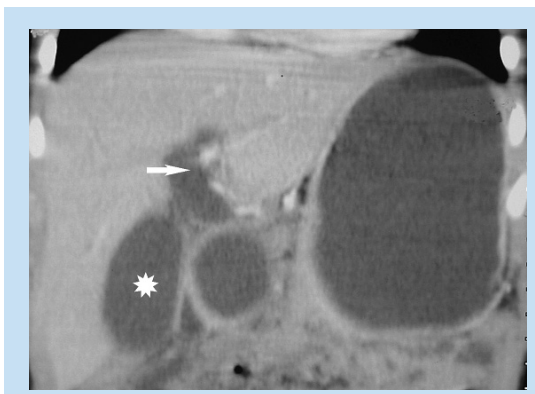

Fig.2b. Coronal reconstructions confirm the well-marginated pancreatic cyst separated from the dilated common duct (arrow) and gallbladder (star).

The differential diagnosis considered included choledochal cyst, congenital cyst, pseudocyst, cystadenoma and hydatid disease. In view of the symptoms, the patient was operated on and the cyst removed. Histopathological examination revealed flimsy translucent membranous material confirmed on microscopy to be consistent with echinococcal disease.

\section{Discussion}

Pancreatic cysts are uncommon in children. ${ }^{7}$ Cysts may be congenital (i.e. developmental) or acquired. The latter includes pseudocysts, retention cysts, duplication cysts, neoplastic cysts and parasitic cysts. Choledochal cysts, although not arising from the pancreas, are intimately related and may sometimes be difficult to distinguish from pancreatic cysts on imaging studies.

Hydatid disease in humans begins with ingestion of eggs, followed by penetration of the gut wall by the embryo and entrance 
into the portal circulation. This explains the high frequency of liver and lung involvement. The liver is involved in approximately $75 \%$ of cases, with lung involvement in $15 \% .{ }^{8}$ It is postulated that possible sources of infestation in isolated pancreas involvement include hematogenous dissemination, local spread via the pancreatobiliary ducts, and peripancreatic lymphatic invasion. ${ }^{3}$

Several classification schemes have been proposed based on the cyst appearance. $^{8} \quad$ Lewall $^{9}$ proposed a four-stage pathology-based classification scheme. The initial stage or type I cyst is a fluid-filled cystic structure with 3 layers: the outermost pericyst composed of modified host cells and fibrous tissue, the middle laminated membrane and the thin inner germinal membrane. A type II cyst has daughter cysts and/or matrix. When the hydatid fluid is replaced by formed elements the lesion dies and eventually becomes calcified and biologically inert, forming a type III lesion. Complications of hydatid disease including rupture and secondary infection are classified as type IV cysts. Three subtypes of rupture are described, viz. contained rupture, communicating rupture and direct rupture with peritoneal spill. ${ }^{10}$

On ultrasound, the appearance of visceral hydatid disease varies according to the stage. ${ }^{11}$ The cyst wall may be a thin welldefined echogenic rim but in certain cases double echogenic lines separated by a central hypoechoic layer have been described. ${ }^{8}$ Internal architecture is also variable. In this case a unilocular anechoic lesion was seen but internal septa, floating membranes, daughter cysts and echogenic hydatid sand have also been described. ${ }^{11}$ The latter creates a snowstorm appearance when the patient is turned over and scanned immediately. ${ }^{10,11}$ Multilocular cysts may manifest with a cyst within a cyst appearance ${ }^{12}$ or a honeycomb pattern with multiple septa, each septum representing the wall of the daughter cyst. Curvilinear cyst wall and internal calcification is also seen as a hyperechoic contour with acoustic shadowing. ${ }^{11}$

CT may display the same findings as ultrasound. The cyst fluid is usually of water attenuation unless complicated by super-added infection. The cyst wall is usually well defined except in super-added infection, with variable nonspecific contrast enhancement. ${ }^{8}$ The classic 'wheel spoke' appearance described on ultrasound is also seen on CT scanning (Fig. 3). ${ }^{11}$

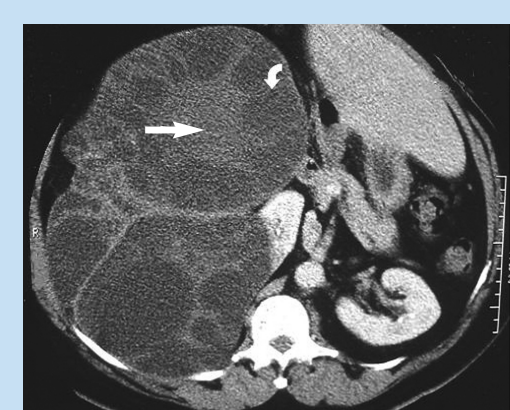

Fig.3. Enhanced CT scan of the abdomen in a different patient showing the typical 'wheel spoke' appearance of a type II cyst in the right kidney. The daughter cysts (curved arrow) are of lower attenuation than the mother cyst and are arranged along the periphery. The mother cyst has a matrix with a density of 35 HU (arrow).

Review of the 5 previously described paediatric pancreatic hydatid cysts, confirmed unilocular cysts with no specific distinguishing features in 3 instances. ${ }^{2,3,6}$ The other 2 cases had more characteristic type II features. ${ }^{4,5}$

In conclusion, the appearance of a cystic mass with an undulating membrane, as with contained rupture, ${ }^{10}$ or of multiple degenerating daughter cysts within the mother cyst ${ }^{9,12}$ may alert the clinician to the possibility of pancreatic hydatid disease.
On the other hand, radiologic examinations alone may not be sufficient to diagnose primary pancreatic hydatid disease and serological tests may be necessary. In our case, both US and CT were useful in diagnosing the cystic mass in the head of the pancreas, but were unhelpful in lesion characterisation. The radiologist should always strive to make the diagnosis of hydatid disease as direct rupture has great clinical significance, including anaphylaxis, hydatid dissemination and secondary bacterial infection. ${ }^{10}$

\section{References}

1. Kattan YB. Hydatid cysts in the pancreas. BMJ 1975; 4: 729-730.

2. Barrera MC, Villanua J, Barrena JF, et al. Pancreatic hydatid disease. Pediatr Radiol 1995; 25: 169-170.

3. Brown RA, Millar AJW, Krige JEJ, et al. Hydatid cyst of the pancreas - a case report in a child. Eur J Pediatr Surg 1995; 5: 121-124.

4. Hicsonmez A. Hydatid cysts in childhood: analysis of 208 cases. Progress in Pediatric Surgery 1982; 15: 87-94.

5. Ismail K, Haluk GI, Necati O. Surgical treatment of hydatid cysts of the pancreas. Int Surg 1991; 76: 185-188.

6. Arikan A, Sayan A, Erikci VS. Hydatid cyst of the pancreas: a case report with 5 years' follow-up. Pediatr Surg Int 1999; 15: 579-581.

7. Rowe MI, O'Neill JA, Grosfeld JL, et al. Essentials of Pediatric Surgery. St. Louis: Mosby Year Book, 1995: 668

8. Pedrosa I, Saiz A, Azzarzalo J, Ferreiros J, Pedrosa CS. Hydatid disease: radiological and pathologic features and complications. Radiographics 2000; 20: 795-817

9. Lewall DB. Hydatid disease: biology, pathology, imaging and classification. Clin Radiol 1998; 53: 863-874.

10. Dahniya MH, Hanna RM, Ashebu S, et al. Pictorial review The imaging appearances of hydatid disease at some unusual sites. Br J Radiol 2001; 74: 283289.

11. Polat P, Kanarci M, Alper F, Suma S, Koruyucu MB, Okur A. Hydatid disease from head to toe. Radiographics 2003; 23: 475-494.

12. Morton PCG, Terblanche JT, Bornman PC, Tyrrell JCH. Obstructive jaundice caused by intrapancreatic hydatid cyst. Br J Surg 1981; 68: 474-476. 